\title{
Cancer et traduction des messagers (suite)
}

En 1990, nous rapportions que l'hyperexpression du facteur d'initiation de la traduction eIF-4E était susceptible de transformer des fibroblastes murins en culture (lignée $\mathrm{NIH} 3 \mathrm{~T} 3)\left(\mathrm{m} / \mathrm{s} n^{\circ} 7\right.$, vol. 6, p. 688). L'équipe de Nahum Sonenberg, de l'université McGill à Montréal (Québec), déjà à l'origine de ces premiers résultats, vient maintenant de démontrer qu'un semblable résultat pouvait être obtenu en interférant, dans les cellules NIH 3T3, avec l'activité de protéine kinases contrôlant la fonction du facteur d'initiation eIF- $2 \alpha$ [1]. Il existe, chez les mammifères, deux protéine kinases susceptibles d'inactiver eIF- $2 \alpha$, et donc d'inhiber la traduction des messagers : HCR (heme control repressor) et dsRNA-PK (double stranded RNA-stimulated protein kinase). Le HCR intervient dans le contrôle de la traduction par l'hème alors que la dsRNA-PK est stimulée par les ARN double brins et par les interférons. L'inhibition traductionnelle ainsi induite par les interférons intervient dans les activités antivirale et anticancéreuse de ces cytokines. La comparaison des séquences protéiques du HCR, des dsRNA-PK murine et humaine et de la kinase de la levure spécifique de eIF-2 $\alpha$ montre l'existence d'un hexapeptide interne absolument conservé dans ces quatre molécules. Cette conservation suggérant un rôle fonctionnel important, Koromilas et al. ont créé une délétion de cette région et transféré un vecteur d'expression commandant la synthèse de la forme ainsi mutée de la dsRNA-PK humaine dans les cellules NIH 3T3 [1]. La synthèse de la dsRNA-PK mutante dans les cellules en culture bloque la phosphorylation d'eIF- $2 \alpha$, probablement par inhibition de l'activité de la kinase endogène (effet trans-dominant). Le résultat est une transformation maligne, bien visible phénotypiquement en culture et tumorigène chez la souris nude (lignée de souris immunodéprimées). La dsRNA-PK normale semble donc relayer l'effet anti-oncogénique de l'interféron, son inactivation se révélant être oncogénique dans des systèmes expérimentaux de cellules en culture.
In vivo, il se pourrait également que fût impliqué un tel système d'" antioncogène " agissant au niveau traductionnel: le gène dsRNA-PK a été trouvé muté, codant pour une protéine kinase inactive, dans un lymphome murin (cité en [1]). Comme nous le discutions en $1990\left(\mathrm{~m} / \mathrm{s} n^{\circ} 7\right.$, vol. 6, p. 688), les mécanismes oncogéniques d'une dérépression traductionnelle restent hypothétiques. Beaucoup de messagers codant pour des facteurs de croissance semblent être contrôlés au niveau traductionnel, ce dont témoigne la longueur de leur région 5' non codante et l'existence à ce niveau de nombreux motifs présomptifs ou avérés d'initiation de la traduction [2]. Se pourrait-il qu'une dérépression anormale des systèmes de traduction permît de surmonter l'atténuation physiologique de la traduction de ces messagers, provoquant alors la transformation cellulaire?

Outre les travaux de l'équipe de Sonenberg, des résultats récents de chercheurs japonais de Kyoto et Osaka militent en faveur de cette hypothèse [3]. Ces chercheurs ont en effet cloné un $\mathrm{ADNc}$ capable de conférer à des cellules résistantes aux agents transformants un phénotype sensible. La séquence de ce clone permit de l'identifier à l'ADNc codant pour le facteur d'élongation EF-1 $\alpha$ qui est fortement et constitutivement synthétisé dans les cellules d'origine de l'ADNc cloné. Ce résultat est tout particulièrement intéressant car il permet de relier transformation et sénescence : $\mathrm{EF}-1 \alpha$ est diminué dans les cellules de mammifères et les drosophiles sénescentes et chez cet insecte, le transfert d'un gène en commandant la synthèse accrue augmente la longévité [4].

A.K

1. Koromilas AE, Roy S, Barber GN, Katze MG, Sonenberg N. Malignant transformation by a mutant of the IFN-inducible ds RNAdependent protein kinase $1992 ; 257: 1685-9$. 2. Prats AC, Vagner S, Prats H, Amalric F. aisacting elements involved in the alternative translation initiation process of human basic fibroblast growth factor mRNA. Mol Cell Biol 1992; 12 4796-805
3. Tatsuka M, Mitsui $H$, Wada M, Nagata A, Nojima $H$, Okayama $H$. Elongation factor- $1 \alpha$ gene determines susceptibility to transformation Nalure 1992 ; 359 : 333-6.

4. Shepherd JCW, Waldorf U, Hug P, Gehring WJ. Fruit flies with additional expression of the elongation factor EF-1 $\alpha$ live longer. Proc Nall Acoc Sci USA 1989 ; 86 : 7520-1.

\section{口 BRÈVE}

Diabète à hérédité maternelle et mutations mitochondriales (suite). Certains diabètes à hérédité maternelle sont d'origine mitochondriale : $\mathrm{m} / \mathrm{s}$ a rapporté récemment $\left(n^{\circ} 6\right.$, vol. 8 , p. 599) l'histoire d'une famille dont les femmes transmettaient à leurs descendants une délétion de $10,5 \mathrm{~kb}$ de l'ADNmt, accompagnée de diabète et de surdité. C'est maintenant une famille néerlandaise dans laquelle un diabète de type II (non insulinodépendant) et une surdité sont liés à une mutation de l'ADNmt [1]. Il ne s'agit plus d'une délétion, mais d'une mutation ponctuelle en position 3243 , dans le gène de l'ADN de transfert de la leucine. Seuls sont affectés les descendants, des deux sexes, des femmes atteintes. La proportion de la forme mutée est variable d'un sujet à un autre. Ce qui peut paraître surprenant, c'est que la mutation 3243 était déjà bien connue. Elle est présente (voir $\mathrm{m} / \mathrm{s}$ $n^{\circ} 2$, vol. 7, p. 172) chez la plupart des malades atteints du syndrome neuromusculaire appelé MELAS, dont cette famille est indemne, et parfois dans des syndromes oculaires du type ophtalmoplégie externe. On sait que, de même $\left(\mathrm{m} / \mathrm{s} n^{\circ} 7\right.$, vol. 5, p. 459), la mutation la plus fréquente de l'ADNmt dite " mutation commune ", qui provoque le plus souvent des syndromes musculaires, peut aussi donner des signes sanguins et pancréatiques du syndrome de Pearson. Il reste donc aux chercheurs à comprendre pourquoi, selon les familles, une même lésion moléculaire peut conduire à des troubles cliniques aussi divers.

[1. Van den Ouweland JMW, et al. Nature Genet 1992 ; 1 : 368-71.] 


\section{aRÈVES $\square \square$}

Des récepteurs minéralocorticoïdes présents dans le cœur et les vaisseaux. M. Lombès et al. [1] (Inserm U. 33, U. 36, U. 241, le Kremlin-Bicêtre et Paris, France) ont démontré la présence de récepteurs minéralocorticoïdes (RM) dans le cœur et les vaisseaux. En utilisant un anticorps monoclonal anti-idiotypique qui interagit avec le domaine de liaison du RM, les auteurs ont localisé les RM dans les myocytes, les cellules endothéliales et probablement certains fibrocytes de l'oreillette gauche. De même, des RM ont été trouvés dans les cellules endothéliales et les cellules musculaires lisses de l'aorte, et dans les myocytes du ventricule gauche cardiaque, mais à un degré moindre que dans les oreillettes. La fixation est encore plus faible dans les artères de plus petit calibre. Des expériences de compétition ont établi la spécificité de la fixation. Les récepteurs minéralocorticoïdes cardiovasculaires ont les mêmes propriétés biochimiques que les récepteurs rénaux, mais leur quantité est environ deux fois moindre que dans le rein (qui est le site principal d'action de l'aldostérone). Le RM cardiaque, comme les autres récepteurs stéroïdes, est associé à la protéine de choc thermique, hsp 90 . La présence des RM suggère une action directe de l'aldostérone sur le cœur et les vaisseaux, mais celle-ci reste à démontrer chez l'animal normal et dans des circonstances pathologiques.

[1. Lombès M, et al. Circulat Res $1992 ; 71: 503-10$.]

口- Anomalie extrinsèque de la migration des neuroblastes intestinaux dans un modèle murin de mégacôlon. La mutation Is de la souris est considérée comme un modèle du mégacôlon chez l'homme (maladie de Hirschsprung). Dans les deux affections, le côlon, et surtout sa partie terminale, est dépourvu de cellules ganglionnaires mésentériques qui sont normalement dérivées du tube neural au niveau des somites 1 à 7 et colonisent la totalité de l'intestin, depuis sa partie proximale jusqu'à sa partie la plus caudale (c'est-à-dire le rectum). Kapur et al., du laboratoire de R. D. Palmiter (Seattle, WA, USA), a créé des souris transgéniques dans lesquelles le gène rapporteur $L a c Z$ est placé sous le contrôle des séquences régulatrices du gène de la dopamine $\beta$ hydroxylase. Dans les souris normales, ce transgène s'exprime au niveau de l'intestin antérieur chez l'embryon murin de 9,5 jours, l'expression progressant vers la partie la plus distale, qui est atteinte au $13,5^{\mathrm{c}}$ jour. Chez des souris homozygotes Is/Is, cette progression s'arrête brutalement au niveau de la valvule iléo-cœcale. La colonisation du côlon proximal et moyen reprend ensuite, mais de façon retardée et irrégulière. Le rectum n'est jamais colonisé. Par conséquent, il semble que la mutation Is à l'état homozygote entraîne un défaut extrinsèque perturbant la migration des neurones mésentériques vers la partie la plus distale du côlon. La nature de ce défaut n'est pas connue. On peut faire l'hypothèse d'une anomalie de l'expression d'une molécule d'adhérence dont l'interaction serait indispensable à cette migration des cellules nerveuses mésentériques. [1. Kapur RJ, et al. Development 1992 ; 116 : 167-75.]

Apoptose, transformation, $b c l-2, m y c$ et $\mathrm{p} 53$. Le proto-oncogène $b c l-2$ code pour une protéine intégrale de la membrane mitochondriale et agit en inhibant la mort cellulaire programmée, c'est-à-dire l'apoptose. Il coopère avec l'oncogène $c$-myc dans la genèse de lymphomes $\left(\mathrm{m} / \mathrm{s} n^{\circ} 1\right.$, vol. 7, p. 88 et $n^{\circ} 4$, vol. 8, p. 392). L'oncogène $c$-myc est, de façon paradoxale, un inducteur d'apoptose dans certaines conditions d'asynchronisme dans la stimulation de la prolifération, par exemple lorsqu'une expression de $c-m y c$ est obtenue dans des cellules privées de sérum et de facteurs de croissance $\left(\mathrm{m} / \mathrm{s} n^{\circ} 6\right.$, vol. 8 , p. 586). Le rapprochement de ces deux informations rendait très proba- ble le mécanisme que viennent d'identifier deux équipes, l'une anglaise - de l'ICRF, Londres [1] - et l'autre américaine - de La Jolla (CA) [2]. Ces deux équipes montrent, en effet, que l'induction de l'apoptose par $c-m y c$ dans des conditions non optimales de croissance cellulaire (absence de sérum, présence de substances anti-mitotiques, déplétion en IL-3 de lignées exigeant cette cytokine pour leur croissance) est bloquée par l'expression de bcl-2. L'équipe anglaise de G. I. Evan fait également une observation intéressante concernant le rôle de $c-m y c$ dans la sensibilité de cellules aux médicaments anticancéreux. La protéine Myc serait responsable d'une apoptose induite par ces substances qui, de par leur effet antiprolifératif, créeraient, en présence d'une expression de $c-m y c$, le même asynchronisme dans les systèmes de régulation de la prolifération cellulaire que la privation de sérum. L'hyper-expression de $b c l-2$, permettant à ces cellules traitées par les antimitotiques d'échapper à l'apoptose induite par $c-m y c$, serait donc un des mécanismes de la résistance aux anticancéreux. Très récemment, un autre mécanisme contrôlant potentiellement la sensibilité des cellules à des antimétabolites a été élucidé : il s'agit de la protéine p53. En effet, p53 semble capable de bloquer la division cellulaire de cellules dont l'ADN est endommagé, alors que ce contrôle est perdu dans des cellules dépourvues de p53 ou, peut-être, contenant une protéine p53 mutée [3, 4]. Dans ce cas, la persistance des proliférations en présence des antimitotiques s'accompagnerait de multiples réarrangements chromosomiques, amplifications et autres anomalies susceptibles de conduire à la sélection clonale de cellules malignes de plus en plus agressives.

[1. Falidi A, et al. Nature $1992 ; 359$ : 554-6.]

[2. Bissonnette RP, et al. Nature 1992 ; 359 : 552-4.]

[3. Livingstone LR, et al. Cell 1992 ; 70 : 923-35.]

[4. Yin Y, et al. Cell 1992; 70 : 937-48.] 
Nos enfants sont des génies ! Dans un article de la revue Nature [1], Karen Wynn décrit les aptitudes arithmétiques des nourrissons. Devant des nourrissons de cinq mois, l'auteur plaçait d'abord une poupée, qui était ensuite masquée par un écran. L'expérimentateur rajoutait alors ostensiblement une deuxième poupée derrière l'écran. Mais subrepticement cette deuxième poupée pouvait être retirée, puis, l'écran une fois enlevé, les réactions du bébé étaient analysées. Dans une deuxième expérience, la situation inverse était proposée aux nourrissons : deux poupées étaient d'abord présentées, puis l'une des poupées était ostensiblement retirée. Comme dans la première expérience, on présentait alors au nourrisson, soit le résultat attendu (une poupée) soit un résultat inattendu (deux poupées). Il est connu que la durée du regard porté par un nourrisson sur une situation est allongée lorsque celle-ci est inattendue. Dans les deux groupes, les nourrissons regardaient plus longtemps les résultats faux $(1+1=1$ ou $2-1=2$ ) que les résultats justes $(1+1=2$ ou $2-1=1)$, auxquels ils semblaient donc s'attendre. Mais les nourrissons s'attendaient-ils simplement à une modification du nombre d'objets de départ ou avaientils pu effectuer un vrai calcul ? Cette dernière hypothèse semble être le cas puisque les nourrissons semblaient aussi intrigués par le résultat $1+1$ $=3$. Les enfants semblent donc capables, dès cinq mois, d'effectuer des additions et des soustractions, ce qui suggère, selon l'auteur, que cette aptitude est innée chez l'homme. Cette découverte pose donc une grave question : nos enfants ne risquent-ils pas d'être perturbés par le stupéfiant échange de deux barils de lessive $\mathrm{X}$ contre un paquet de poudre $\mathrm{Z}$ ?

[1. Wynn K. Nature 1992 ; 358 : 749-50.]

Rats transgéniques pour les gènes de la rénine et/ou de l'angiotensinogène humains. Ganten et al. (Heidelberg, Allemagne) ont créé des rats transgéniques ayant intégré dans leur génome le gène entier de la rénine humaine ( $h R E N)$, le gène entier de l'angiotensinogène humain (hAOGEN) ou les deux gènes [1]. Chez les rats TGR (hREN) hétérozygotes, l'expression du transgène est forte dans le rein et notamment dans l'appareil juxtaglomérulaire (là où est produite la rénine endogène du rat) mais est aussi présente dans le poumon, le thymus et l'appareil digestif. La rénine et la prorénine humaines sont détectées dans le plasma des rats transgéniques, et la concentration de rénine humaine est stimulée par la déplétion sodée. Néanmoins, comme la rénine humaine ne réagit pas avec l'angiotensinogène de rat, les taux plasmatiques d'angiotensine I et II (ANG I et II) ne sont pas augmentés ; la pression artérielle de ces animaux est normale. Chez les TGR (hAOGEN) hétérozygotes, les taux plasmatiques d'angiotensinogène humain sont très élevés, mais les taux d'ANG II sont inchangés. L'expression du transgène est forte dans le foie, le rein, le cerveau, le poumon, le cœur et l'appareil digestif; par hybridation in situ, l'expression du transgène se fait dans le foie, dans les hépatocytes, au site connu de synthèse. La perfusion de rénine humaine à ces rats transgéniques entraîne une nette élévation de la pression artérielle, réponse supprimée par l'administration préalable d'un inhibiteur spécifique de la rénine. Enfin, par croisement entre des rats TGR (hREN) et TGR (hAOGEN), des animaux possédant les deux transgènes ont été engendrés; fait remarquable, ces animaux ne deviennent pas spontanément hypertendus. [1. Ganten D, et al. Proc Natl Acad Sci USA 1992 ; 89 : 7806-10.]

p53 et pronostic du cancer du col utérin. Un grand nombre de cancers du col utérin est dû à l'infection par le virus du papillome humain HPV (human papilloma virus). $\mathrm{Ce}$ dernier agit principalement par l'intermédiaire des produits de ses oncogènes $E 6$ et $E 7$. E7 forme un complexe avec la protéine p105 ${ }^{R b}$, produit du gène de susceptibilité au rétinoblastome, alors que $E 6$ se fixe à la protéine p53 dont il induit la dégradation protéolytique. De ce fait, l'infection par HPV suffit à créer un déficit en p53. Cela explique probablement que les cancers du col, positifs pour le génome HPV, ne comportent habituellement pas de mutations des gènes cellulaires p53. En revanche, ces mutations sont extrêmement fréquentes dans les cancers sans relation avec une infection par HPV. T Crook et K.H. Vosden (Londres, GB) [1] viennent de montrer que les métastases ganglionnaires de malades atteints de cancers du col positifs pour l'ADN HPV possédaient des gènes p53 mutés, même lorsqu'aucune anomalie du gène $p 53$ n'était observée au niveau de la tumeur primitive. Ces résultats confirment bien que la mutation de p53 est un événement important de la progression tumorale. Ils expliquent aussi que les cancers négatifs pour l'ADN HPV aient un pronostic plus mauvais que ceux en rapport avec une infection par HPV. Dans le premier cas, en effet, le gène p53 est d'emblée muté, ce qui pourrait correspondre à une malignité initiale d'emblée importante.

[1. Crook T, Vosden K.H. EMBO J $1992 ; 11: 3935-40$.

\section{SOCIÉTÉ DE BIOLOGIE}

Séance du 9 décembre 1992, à $16 \mathrm{~h} \mathrm{30}$, au Collège de France, 11 place M. Berthelot, 75005 Paris, consacrée à la Génétique moléculaire, avec la participation de Mme C. Rayssiguier, MM. D. Anxolabehere, R. Perasso, A. Raisonnier, A. Blanchet, P. Calvaz, J. Ruffie, Y. Coppens, A. Langaney.

Contact: Mlle Faibie, Société de Biologie, Collège de France

Tél. : (1) 44.27.13.40 


\section{BRÈVES ם}

Les lésions moléculaires du diabète insipide néphrogénique. Le diabète insipide, marqué cliniquement par soif et polyurie, est typiquement dû à une anomalie du nonapeptide arginine-vasopressine, dont le gène siège sur le chromosome $20 \mathrm{~m} / \mathrm{s} n^{\circ} 3$, vol. 8, p. 291). On en connaît une deuxième forme liée au nonfonctionnement du récepteur de la vasopressine, appelé V2, concentré surtout dans le tube contourné du rein. Dans le diabète insipide congénital néphrogénique, une affection récessive liée au sexe, le rein ne répond en effet pas à la vasopressine. Une équipe de Houston, (TX, USA) vient de cloner le récepteur humain à partir d'une banque d'expression $\left(\mathrm{m} / \mathrm{s} n^{\circ} 7\right.$, vol. 8, p. 727) [1]. L'ADNc obtenu du rein code pour une protéine de 371 acides aminés, dont la séquence fait prévoir l'existence de sept fragments transmembranaires. Sa structure est celle des récepteurs couplés à une protéine $\mathrm{G}$. Son gène a été localisé sur le chromosome $\mathrm{X}$ en Xq28-qter. Le messager d'environ $2 \mathrm{~kb}$ est concentré essentiellement dans le rein. Ainsi se trouve assurée la réabsorption de l'eau. La même équipe, associée à des chercheurs canadiens, a étudié un malade, actuellement âgé de 34 ans, ne présentant depuis l'enfance aucune réponse à la vasopressine [2]. L'analyse de la région codante de l'ADNc montra une délétion d'une guanosine, à l'intérieur d'une séquence qui en compte six consécutives (nucléotides 733-738). Cette délétion modifie le codon 246, change le cadre de lecture et aboutit à une terminaison prématurée en position 270. Un frère normal ne possédait pas la délétion, alors que la mère la présentait à l'état hétérozygote. Dans une autre famille, on trouva non une délétion, mais une mutation ponctuelle Ala $132 \rightarrow$ Asp, dans le troisième segment transmembranaire ; dans ce cas également la mère était hétérozygote. Il semble s'agir du premier exemple d'une anomalie génétique d'un récepteur hormonal couplé à une protéine $\mathrm{G}$ (alors qu'on connaît des troubles d'un photorécepteur couplé à une protéine $\mathrm{G}$, avec comme résultat une rétinite pigmen- taire) [3]. Le gène du diabète insipide congénital néphrogénique a pu être localisé par les mêmes auteurs [2] dans la même zone que celui du récepteur V2 et il est probable qu'il y a identité entre les deux. Il reste à savoir si d'autres malades atteints de ce syndrome clinique présentent eux aussi des anomalies du récepteur V2.

[1. Birnbaumer $\mathrm{M}$, et al. Nature $1992 ; 357$ : 333-5.]

[2. Rosenthal W, et al. Nature 1992 ; 359: 233-5.]

[3. Sung CH, et al. Proc Natl Acad Sci USA 1991; 88 : 8840-4.]

Recherches des mutations du précurseur de la protéine $\beta$ amyloïde dans un grand nombre de cas familiaux et sporadiques de maladie d'Alzheimer. Dans une nouvelle récente $\left(n^{\circ} 8\right.$, vol. $\left.8, p .866\right), \mathrm{m} / \mathrm{s}$ $s$ 'efforçait de dénombrer les lésions moléculaires du précurseur APP de la protéine $\beta$-amyloïde, qui peuvent accompagner une maladie d'Alzheimer ou des affections voisines. Ce type d'approche n'indique pas la fréquence des lésions de l'APP dans l'ensemble des maladies d'Alzheimer, familiales ou sporadiques. C'est à cette tâche que s'est attelé un groupe important de chercheurs d'Amérique du Nord (21 auteurs). Leur point de départ [1] fut l'étude d'une grande famille qui semblait montrer une liaison avec le chromosome 21 . Le séquençage de toute la région codante du gène $A P P$ n'a montré aucune mutation. On a ensuite recherché une liaison génétique à l'APP dans 25 familles à début précoce ou tardif, sans trouver un seul lod score positif. On a séquencé les exons 16 et 17 (qui couvrent le domaine $\beta$-amyloïde de l'APP) dans 30 familles et chez 11 cas sporadiques,; enfin 56 familles et 81 cas sporadiques ont été criblés pour la mutation la plus fréquente APP $717 \mathrm{Val} \rightarrow$ Ile. Aucune réponse positive n'a été obtenue. Les auteurs concluent que les mutations du gène de l'APP ne rendent compte que d'une très faible proportion des maladies d'Alzheimer, même familiales. [1. Tanzi RE, et al. Am J Hum Genet 1992 ; 51 : 273-82.] 
Une anomalie moléculaire dans la protoporphyrie. Le déficit en ferrochélatase provoque l'accumulation de protoporphyrine, marquée par une photosensibilité et des troubles hépatobiliaires. Le séquençage de l'ADNc a montré chez un malade une mutation (Phe $\rightarrow$ Ser) à l'extrémité Cterminale de la protéine. Le gène a pu être localisé en 18q21-3 par hybridation in situ. D'autres malades, toutefois, ne portaient pas cette mutation.

[Brenner DA, et al. Am J Hum Genet 1992 ; 50 : 1203-10.]

Le gène de l'acétylcholinestérase humaine vient d'être localisé par une équipe californienne sur le chromosome 7 en $7 q 22$ et semble bien être unique.

[Getman DK, et al. Am J Hum Genet 1992 ; 51 : 170-7.]

Rétinite pigmentaire récessive par anomalie de la rhodopsine. Les mutations de la rhodopsine provoquent des rétinites pigmentaires à hérédité autosomique dominante $(\mathrm{m} / \mathrm{s}$ $n^{\circ} 1$, vol. 8, p. 82). Rosenfeld et al. (Boston, MA, USA) viennent de décrire [1] une " mutation zéro" (null mutation), par apparition d'un codon non-sens en position 249. Les hétérozygotes sont cliniquement sains et seuls les homozygotes ont une rétinite. L'atteinte d'un même gène peut donc provoquer des maladies récessives ou dominantes; de plus, dans certains cas, mieux vaut pas de protéine du tout, pour un hétérozygote, qu'une protéine déf ectueuse [2].

[1. Rosenfeld PJ, et al. Nature Genet 1992 ; 1 : 209-13.]

[2. McInnes KR, Bascom RA. Nature Genet 1992 ; 1 : 155-7.]

Le gène qui code pour le récepteur $\alpha$ du facteur stimulant des granulocytes et des macrophages (CSF2RA) est localisé chez l'homme sur la région pseudo-autosomique commune aux deux chromosomes sexuels. Chez la souris, il siège sur un autosome, le chromosome 19. C'est le premier exemple d'un gène porté par un chromosome sexuel chez l'homme et par un autosome dans $\mathrm{m} / \mathrm{s} n^{\circ} 9 \mathrm{vol} .8$, novembre 92 une autre espèce de mammifères, à l'exception (m/s $n^{\circ} 3$, vol. 8, p. 259) des mammifères primitifs, marsupiaux et monotrèmes.

[Disteche CM, et al. Nature Genet $1992 ; 1$ : 333-5.]

Le déficit en glucose-6-phosphate déshydrogénase du type A atteint des millions d'individus d'origine africaine. Il comporte deux mutations par rapport à l'enzyme sauvage du type B : la mutation 126 Asn-Asp caractéristique de la forme A, sur laquelle s'est greffée une seconde mutation Met 68-Val. Une équipe britannique a analysé in vitro l'effet de chacune de ces mutations. Elle a conclu que la présence simultanée des deux était nécessaire pour provoquer l'instabilité moléculaire caractéristique de la G6PD A.

[Town M, et al. Hum Mol Genet $1992 ; 1: 171-4$.

La myopathie des ceintures est génétiquement hétérogène. Le terme de myopathie des ceintures englobe plusieurs syndromes cliniques. Une forme, fréquente à la Réunion, avait été localisée en 1991 sur le bras long du chromosome 15. La preuve de son hétérogénéité génétique vient d'être apportée par une équipe francoaméricaine qui, dans une famille, a montré le gène lié au chromosome 5 en 5q22.3-31-3.

[Speer MC, et al. Am J Hum Genet 1992 ; 50 : 1211-7.]

Une délétion de huit acides aminés a été découverte dans le gène de la protéine prion à l'état hétérozygote chez une personne présentant une démence de l'âge mûr [1]. Cette lésion s'ajouterait à la liste des anomalies pathogènes des prions $(\mathrm{m} / \mathrm{s}$ $n^{\circ} 6$, vol. 7, p. 626). Elle doit cependant être interprétée avec prudence car de telles délétions de $24 \mathrm{pb}$ ont été signalées chez des sujets normaux $[2,3]$.

[1. Diedrich JF, et al. Hum Mol Genet 1992 ; 1 : 443-4.]

[2. Laplanche JL, et al. Nucleic Acids Res 1990 ; 18 : 6745.]

[3. Vnencak-Jones CL, Phillips JA. Am J Hum Genet 1992 ; 50 : 871-2.]

Jean-Claude Dreyfus 